\title{
Analysis of the Porous Structures from Laser Powder Bed Fusion Additive Manufacturing
}

\author{
Chang Jiang WANG ${ }^{\mathrm{a}, 1}$, Kevin HAZLEHURST ${ }^{\mathrm{b}}$, Arun ARJUNAN ${ }^{\mathrm{c}}$ and Lida Shen ${ }^{\mathrm{d}, 1}$ \\ ${ }^{a}$ Department of Engineering and Design, University of Sussex, Brighton BN1 9RH, UK \\ ${ }^{\mathrm{b}}$ Conflux Technology Pty Ltd, 75 Pigdons Road, Waurn Ponds, VIC 3216, Australia \\ ${ }^{\mathrm{c}}$ School of Engineering, University of Wolverhampton, Telford, TF2 9NT, UK \\ ${ }^{\mathrm{d}}$ College of Mechanical and Electrical Engineering, Nanjing University of Aeronautics \\ and Astronautics, Yu Dao Street, Nanjing, 210016, PR China
}

\begin{abstract}
Open and closed porous structures with lattice and honeycomb geometry can be built using laser powder bed fusion additive manufacturing processes. The porous structures can be used to tailor the mechanical properties of a component or provide other functionality, such as for bone ingrowth in medical implants. Porous structures were created and analysed in this paper both physically and using finite element modelling. It was found that the accuracy of the built parts was reasonable and within the manufacturing processes general tolerance of $+/-50 \mu \mathrm{m}$. However, it was noticeable that the corners of the square shape pores were naturally filleted by the manufacturing process. The finite element model was developed using ANSYS software, stress concentrations were observed in the porous structures under loading. In addition to this, fragments of the material were present on the internal surfaces of the pores, which were formed from partially melted powder particles.
\end{abstract}

Keywords. additive manufacturing, porous structure, finite element analysis.

\section{Introduction}

Additive Manufacturing (AM) technologies such as Direct Metal Laser Sintering (DMLS), Selective Laser Sintering (SLS) and Electron Beam Melting (EBM) have been utilised for orthopaedic applications $[1,2,3]$. It has been observed that AM has the potential to produce customised, lightweight, functionally graded implants with tailored mechanical properties to reduce the stress shielding phenomenon whilst also providing a surface topography that will encourage biological fixation at the bone-implant interface [4].

Titanium alloys were originally developed primarily for use in the aerospace industry and are now frequently utilised for biomedical applications. Ti-6Al-4V is often used for femoral, tibial and humeral components in joint arthroplasty due to its biocompatibility and because it has mechanical properties that exceed cobalt-chromium and stainless steel alloys in terms of implant suitability. In particular, the property of

${ }^{1}$ Corresponding Authors, Department of Engineering and Design, University of Sussex, BN1 9RH, UK. E-mail: c.j.wang@sussex.ac.uk; ldshen@nuaa.edu.cn. 
interest is that the elastic modulus of Ti-6Al-4V is relatively low when compared to the other mentioned alloys and hence, closer to the elastic modulus of human bone.

AM can build open and closed porous structures with lattice and honeycomb type geometry. Therefore, the unit cell approach is a common method that has been used to design bone-like structures. The unit cell approach is where a cell with regular geometry is designed in 3D CAD software and is then geometrically defined in a sequence to create a 3D porous structure. Possible structures based upon the unit cell approach include cubes, truncated octahedrons, truncated cubes, truncated cuboctahedrons, triangular prisms, rectangular prisms, octagonal prisms and rhombic dodecahedrons. A possible negative factor associated with the unit cell approach is when fitting the unit cells to a curved surface, as errors can be induced in the design process. Either leaving unit cells exposed from the component surface or leaving unstable single struts exposed internally. Both could have a detrimental effect on the mechanical strength of such a component.

The problem of downward-facing or overhanging surfaces is inherent with AM processes because the metal powder cannot support itself during the build process. A general recommendation is that any surface with an inclination angle of less than $45^{\circ}$ from the machine bed should be supported by sacrificial structures. This factor is of particular concern with building porous structures where the orientation of individual unit cells can be very complex, and the removal of support structures would be too cumbersome. Therefore, particular detail needs to be applied to the design process for building self-supporting lattice structures of high quality with the required structural integrity.

Many researchers have studied the mechanical properties of porous structures manufactured from AM. Arjunan et al. investigated the stiffness, strength, permeability and stress concentration of six scaffold architectures with 68.46 to $90.98 \%$ porosity [5]. The results showed that the pore shape influenced permeability, stiffness, strength and the stress concentration factor of Ti-6Al-4V bone scaffolds. Liang et al. studied the geometric accuracy of the struts manufactured from the Laser Powder Bed Fusion (LPBF) process. The struts possessed a thickness of $0.4 \mathrm{~mm}$ to $1.4 \mathrm{~mm}$ and were manufactured in three directions of $0^{\circ}, 45^{\circ}$ and $90^{\circ}$ with reference to the horizontal building plate [6]. The authors found that the built struts were larger than the designed thickness, and the struts of $0^{\circ}$ were thicker than the struts of $90^{\circ}$. Zhu et al. conducted a fatigue test on porous scaffolds and found that the fatigue property was greatly affected by the porosity and heat treatment process [7]. However, there is a lack of research work on the stress concentration in the porous structures from additive manufacturing.

This paper will focus on the post-processing of lattice structures manufactured using LPBF and the corner intersections of the struts, to understand the quality of the porous structures.

\section{Methods}

Square pore lattice structures with pore sizes ranging between $0.1 \mathrm{~mm}$ and $0.5 \mathrm{~mm}$ were designed and manufactured. Components were designed using SolidWorks and were sized at $15 \mathrm{~mm} \times 15 \mathrm{~mm} \times 3 \mathrm{~mm}$ to obtain a reasonable build time. The pore and strut sizes of the different designs are listed in Table 1. Two components per design were manufactured so that the as-built structures could be compared with the shot blasted components. The components were manufactured using the EOSINT M270 Xtended 
Direct Metal Laser Sintering machine with standard operating parameters for Ti-6Al-4V. Upon completion of the build, the components were depowdered and removed from the build platform using wire Electron Discharge Machining (EDM). Compressed air was passed through the structures to remove any excess powder. Half of the components were shot blasted using ceramic particles and the remainder of the components were left in their as-built state. The components were examined using the Olympus LEXT optical laser scanning confocal microscope (OLS 3000) to observe the structures at an acceptable scale.

The Finite Element (FE) model of the built structure of Component 7 was created using ANSYS software for stress analysis. The FE mesh of plane element, constraints and horizontal displacement loads are shown in Figure. 1. The Ti-6Al-4V material was modelled as bilinear isotropic with $E=115 \mathrm{GPa}, v=0.3$, yield strength $\sigma_{y}=1000 \mathrm{MPa}$ and tangent modulus $E_{t}=1300 \mathrm{MPa}$.

Table 1. Dimensions and porosity of square pore lattice structures

\begin{tabular}{ccc}
\hline Component & Pore size (mm) & Strut size (mm) \\
\hline 1 & 0.20 & 0.15 \\
2 & 0.25 & 0.11 \\
3 & 0.30 & 0.25 \\
4 & 0.35 & 0.32 \\
5 & 0.40 & 0.45 \\
6 & 0.45 & 0.24 \\
7 & 0.50 & 0.38 \\
\hline
\end{tabular}

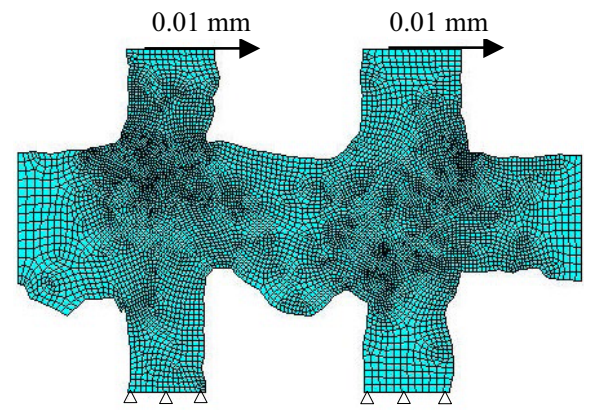

Figure 1. Finite element model of the built struts

\section{Results}

The accuracy of the built parts was reasonable in that it was within the manufacturing processes general tolerance of $+/-50 \mu \mathrm{m}$. However, it was noticeable that the corners of the square shape pores were naturally filleted by the manufacturing process. In addition to this, it can be seen from Figure 2 (a) and (b) that there are fragments of the material present internal to the pores, which are formed from partially melted powder particles. It is normal practice to remove these fragments by using post-processing methods such as shot blasting. However, due to the intricate structures used for these components, some potential issues were identified. It can be seen from Figure 2 (c) that although some of the fragments have been removed there is an issue with the shot blasting media being 
trapped inside the pores. In addition to this, it can be seen from Figure 2 (d) that the shot blasting process can damage the structures, with individual horizontal interconnecting struts being fractured. For clarity, the spherical type regions which are shown in Figure 2 (d) typically represent the remains of vertical struts.

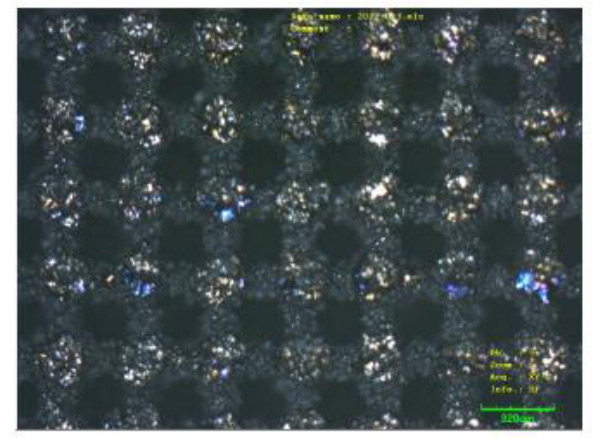

(a)

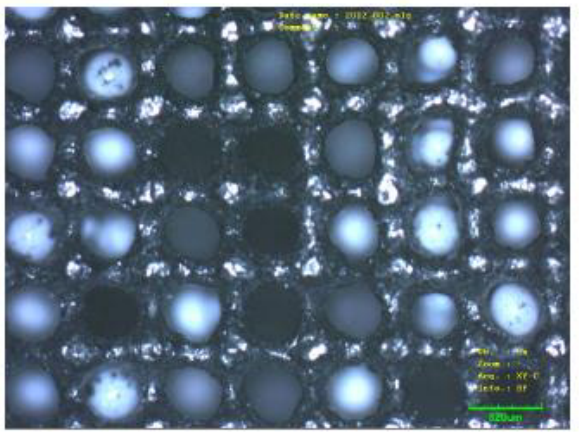

(c)

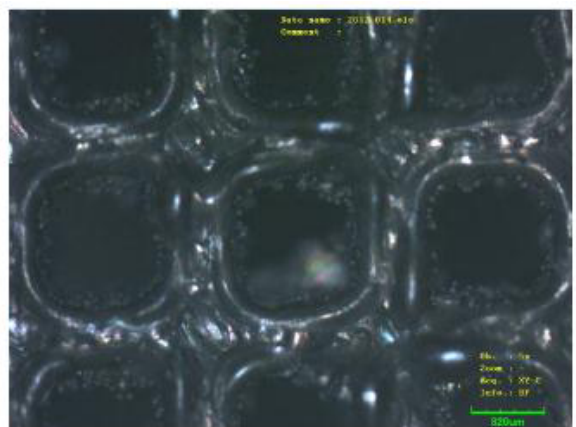

(b)

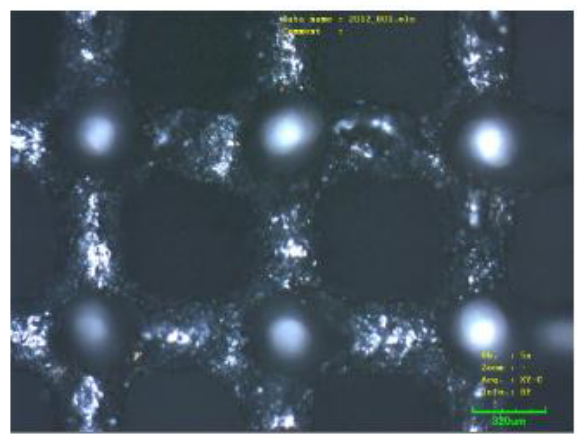

(d)

Figure 2. (a) View in the horizontal plane of the as-built component with $0.25 \mathrm{~mm}$ pore size, (b) View in the horizontal plane of the as-built component with $0.50 \mathrm{~mm}$ pore size, (c) View in the horizontal plane of the shot blasted component with $0.25 \mathrm{~mm}$ pore size, (d) View in the horizontal plane of the shot-blasted component with $0.5 \mathrm{~mm}$ pore size.

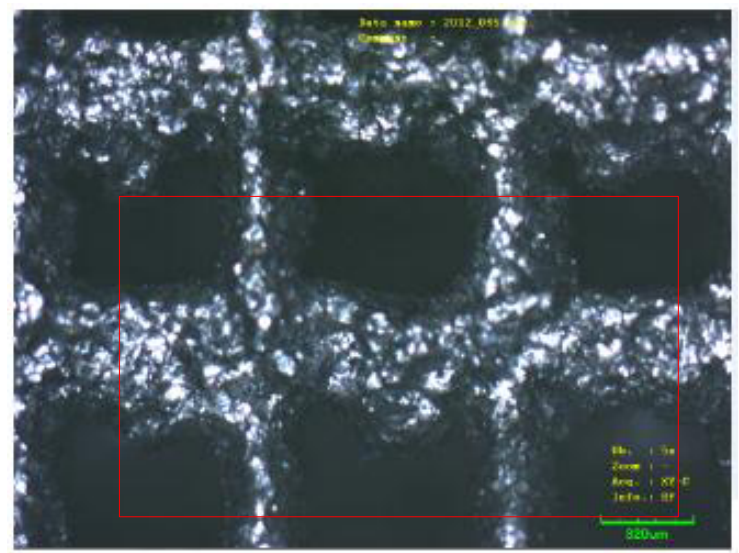

Figure 3. View in the vertical plane of the shot-blasted component with $0.50 \mathrm{~mm}$ pore size. 
Figure 3 shows the problem that the downward-facing surfaces create in that there is what can be described as a "waving effect" of the horizontal struts on the outside faces of the component. It is also evident that this problem is more noticeable as the pore size increases. This could well have a negative effect on the structural integrity of such components and would make an inspection of these internal structures extremely difficult. The connections of the struts vary greatly and are irregular. To assess the effect on structural integrity, the struts shown inside the red box in Figure 3 were modelled for stress analysis. The FE model is shown in Figure 1, mesh sizes are reasonable and converged for the study.

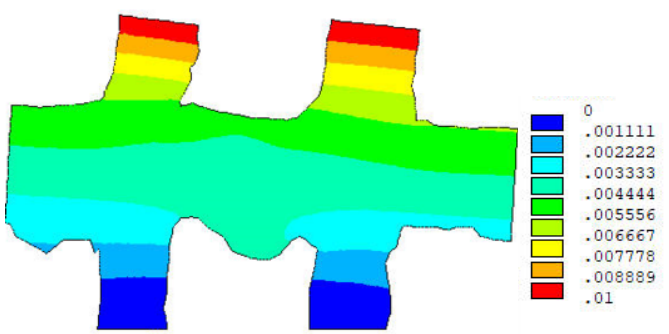

Figure 4. Deformation (mm) of the FE model under horizontal shearing load

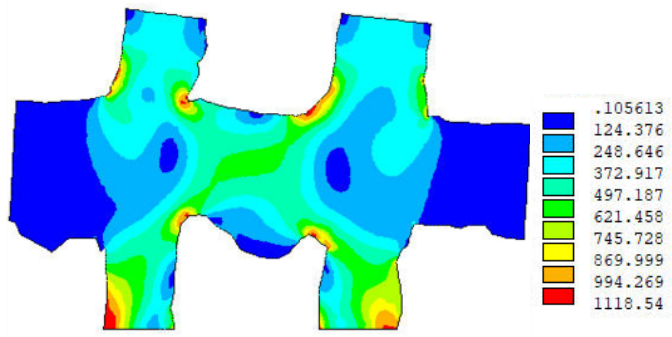

Figure 5. von Mises stress (MPa) distribution on the built struts.

Figure 4 shows the deformation of the struts under the horizontal shearing load of a $0.01 \mathrm{~mm}$ displacement. It can be observed that the struts are deformed non-uniformly. The von Mises stress which is shown in Figure 5 also highlights stress concentrations at the corner intersections.

Mechanical properties of porous structures have been extensively studied by other researchers which can be found in the works by Hazlehurst et al. [8].

\section{Discussion}

Porous structures exhibit a good strength to weight ratio, high surface area and a lower stiffness when compared to solid structures. These structures have been widely incorporated in additive manufactured components, such as heat exchangers and medical implants. In some applications, the mechanical strength and surface finish of the porous structures are important. This paper investigated the production of small struts and it is evident that the thickness of the built struts can be reasonably obtained in the horizontal 
plane. However, some issues arise in creating accurate struts in the vertical plane, due to the introduction of downward-facing surfaces. The quality and consistency of struts in the vertical plane is very difficult to achieve. Consequently, the irregular intersections of the struts could lead to early fractures because of stress concentrations. If the strength of additive manufactured porous structures is considered important, a safety factor should be researched and implemented into the design of such end-use components.

From a biomedical perspective, the issue of unwanted fragments of material or powder particles within the porous structure is unacceptable due to the potential contamination from metal ions that can cause complications to the human body. However, removing the powder particles remains a challenging problem. Dong et al. used electrochemical polishing and chemical etching methods to improve the surface finish of Ti-6Al-4V manufactured by direct metal laser sintering [9]. However, the electrochemical polishing process is not effective on the internal features as too much material was removed.

\section{Conclusion}

It is evident that LPBF can produce intricate porous structures with pore sizes ranging between $0.20 \mathrm{~mm}$ and $0.50 \mathrm{~mm}$. However, it is noticeable that there are factors that need to be considered in terms of removing partially melted fragments/powder and designing self-supporting structures that minimise the problem of downward-facing surfaces.

\section{References}

[1] Espana FLA, Balla VK, Bose S, Bandyopadhyay A. Design and fabrication of CoCrMo alloy based novel structures for load bearing implants using laser engineered net shaping. Mater Sci Eng: C. 2010; 30: 50-57. Available from: https://doi.org/10.1016/j.msec.2009.08.006

[2] Bertol LS, Junior WK, da Silva FP, Aumund-Kopp C. Medical design: Direct metal laser sintering of Ti6Al-4V. Mater Des. 2010; 31: 3982-3988. Available from: https://doi.org/10.1016/j.matdes.2010.02.050

[3] Parthasarathy J, Starly B, Raman S, Christensen A. Mechanical evaluation of porous titanium (Ti6A14V) structures with electron beam melting (EBM). J Mech Behav Biomed Mater. 2010; 3: 249- 259. Available from: https://doi.org/10.1016/j.jmbbm.2009.10.006

[4] Hazlehurst K, Wang C, Stanford M. A numerical investigation into the influence of the properties of cobalt chrome cellular structures on the load transfer to the periprosthetic femur following total hip arthroplasty. Med Eng Phys. 2014; 36: 458-466. Available from: DOI: 10.1016/j.medengphy.2014.02.008

[5] Arjunan A, Demetriou M, Baroutaji A, Wang C. Mechanical performance of highly permeable laser melted Ti6Al4V bone scaffolds. J Mech Behav Biomed Mater. 2019; 102: 103517. Available from: https://doi.org/10.1016/j.jmbbm.2019.103517

[6] Liang H, Xie D, Mao Y, Shi J, Wang C, Shen L et al. The size effect on forming quality of Ti-6Al-4V solid struts fabricated via laser powder bed fusion. Metals. 2019; 9: 416. Available from: https://doi.org/10.3390/met9040416

[7] Zhu L, Liang H, Lv F, Xie D, Wang C, Mao Y, et al. Design and compressive fatigue properties of irregular porous scaffolds for orthopedics fabricated using selective laser melting. ACS Biomater Sci Eng. 2021; 7 : 1663-1672. Available from: https://doi.org/10.1021/acsbiomaterials.0c01392

[8] Hazlehurst K, Wang CJ, Stanford M. Evaluation of the stiffness characteristics of square pore CoCrMo cellular structures manufactured using laser melting technology for potential orthopaedic applications. Mater Des. 2013; 51: 949-955. Available from: https://doi.org/10.1016/j.matdes.2013.05.009

[9] Dong G, Marleau-Finley J, Zhao YF. Investigation of electrochemical post-processing procedure for Ti6AL-4V lattice structure manufactured by direct metal laser sintering. Int J Adv Manuf Technol, 2019; 104: 3401-3417. Available from: https://doi.org/10.1007/s00170-019-03996-5 